N. Slavinskaya, J.H. Starcke, M. Abbasi, A. Mirzayeva, U. Riedel, M.

Frenklach, A. Packard, W. Li, Wenyu, J. Oreluk, A. Hedge, Consistent Syngas Chemical Mechanism from Collaborative Data Processing. In: Proceedings. 55th AIAA Aerospace Sciences Meeting, 08.-13.01.2017, Paper AIAA 2017-0837, Grapevine, USA.

The AIAA version of the paper is accessible at http://dx.doi.org/10.2514/6.2017-0837

On the AIAA web page http://www.aiaa.org/content.cfm?pageid=2

the interested reader can find other material published by AIAA 


\title{
Consistent Chemical Mechanism from Collaborative Data Processing
}

\author{
N.A. Slavinskaya ${ }^{1}$, J.H.Starcke ${ }^{2}$, M.Abbasi ${ }^{3}$, A.Mirzayeva $^{4}$, U.Riedel $^{5}{ }^{5}$ \\ German Aerospace Center (DLR), Institute of Combustion Technology, 70569, Stuttgart, Germany \\ M. Frenklach ${ }^{6}$, A. Packard ${ }^{7}$, W. Li ${ }^{8}$, J. Oreluk $^{9}$, A. Hedge ${ }^{10}$ \\ Mechanical Engineering, University of California at Berkeley, Berkeley, CA 94563, USA
}

The Bound-to-Bound Data Collaboration (B2B-DC) module of the automated data-centric infrastructure of PrIMe was used for the systematic uncertainty and data consistency analyses of the $\mathrm{H}_{2} / \mathrm{CO}$ reaction model (73/17) and 118 experimental targets (ignition delay time and laminar flame speed). The performed consistency analysis of the composed dataset identified a set of experimental data that were inconsistent and therefore removed from the dataset for future investigation. The final consistent dataset with $\mathbf{5 7}$ experimental targets and 28 active variables was used for the B2B-DC framework model optimization and analysis on the feasible parameter set. The produced optimized syngas models demonstrated an improved agreement with the studied dataset, as well as with experimental measurements not included in the analysis. The obtained optimized parameter values indicated parameter inadequacy, and the correlation analysis highlighted the direction of possible parameter modifications and model improvement. The initial results demonstrate clear benefits of the PrIMe methods for developing predictive kinetic models.

\section{Introduction}

$\mathrm{T}$ o reliably develop predictive reaction models for complex chemical systems requires integration of large amounts of theoretical, computational, and experimental data collected by numerous researchers. The integration entails assessment of the consistency of the data, validation of models, and quantification of uncertainties for model predictions. This approach to the development of mechanistic reaction models consists of conjecturing the reaction mechanism and comparing the predictions of the constructed model to available experimental observations. Typically, such comparisons result in mixed outcomes: some show a reasonably close agreement and some do not. In the latter case, the apparent inconsistency obtained between the model and the experiment is argued to imply either that the model is inadequate or that the experiment (or, rather, its interpretation) is incorrect. The application of computational modeling demands on models to be accurate, reliable, and first of all, predictive.

In the present paper the Data Collaboration module of cyber-infrastructure PrIme ${ }^{1}$ (Process Informatics Model) was tested for uncertainty prediction and optimization of chemical reaction model. PrIme ${ }^{1}$ is designed for analysis, processing and storage of large amounts of original data using advanced mathematical methods. Here we present preliminary results of the analysis, with a more complete one forthcoming.

Bound-to-Bound Data Collaboration, abbreviated hereafter as B2B-DC, is an optimization-based framework for combining models and experimental data from multiple sources to explore their collective information content. The approach can decisively indicate whether related experimental data are consistent with each other within a specified chemical kinetics model, explore sources of inconsistency, discriminate among differing models, make model interval predictions, and analyze sensitivity of uncertainty propagation. We begin by reiterating some key definitions. $^{1-8}$

\footnotetext{
${ }^{1}$ Senior research fellow, Chemical Kinetics Department, Nadja.Slavinskaya@dlr.de, AIAA Senior Member.

${ }^{2}$ Senior research fellow, Chemical Kinetics Department, JanHendrik.Starcke@dlr.de.

${ }^{3}$ PhD Student, Chemical Kinetics Department, _Mehdi.Abbasi@dlr.de.

${ }^{4}$ PhD Student, Chemical Kinetics Department, Aziza.Mirzayeva@dlr.de

${ }^{5}$ Prof. Head of Chemical Kinetics Department, Uwe.Riedel@dlr.de, AIAA Senior Member

${ }^{6}$ Prof. of University of California, Berkeley, frenklach@, berkeley.edu, AIAA Member.

${ }^{7}$ Prof. of University of California, Berkeley, apackard@berkeley.edu.

${ }^{8} \mathrm{PhD}$ Student of University of California, Berkeley, wenyuli@berkeley.edu.

${ }^{9} \mathrm{PhD}$ Student of University of California, Berkeley, jim.oreluk@berkeley.edu.

${ }^{10} \mathrm{PhD}$ Student of University of California, Berkeley, arun.hegde@berkeley.edu.
} 
Quantities of Interest (QoI) is a collection of experimental observations of physical processes, coupled with respective uncertainties, assessed as lower and upper bounds on the observed values, i.e., $L_{e}$ and $U_{e}$ for each $e$-th QoI. This physical process can be represented by a numerical model, $M(x)$, with prior knowledge on the domain of parameters, thus constraining each $x$ to an interval $\left[x_{\min }, x_{\max }\right]$ and all together to a hypercube $x \in \mathrm{H}$.

A key requirement for B2B-DC is the formulation of a dataset $\mathbf{D}$, which entails creation of dataset units for all QoI, $e=1,2, \ldots$, from experimental observations, common kinetic model, and their uncertainties. The computational model $M(x)$ must produce outputs that are consistent with the reported QoI uncertainties.

Hence additional constraints that the true parameters must satisfy are

$$
L_{e} \leq M(x) \leq U_{e} \quad \text { for all } e
$$

The subset of $\boldsymbol{H}$ satisfying (1) is called the feasible set, $\boldsymbol{\Phi}$, of parameters. $\boldsymbol{\Phi}$ is simply all parameter values that jointly satisfy all of the prior information and are consistent with all model predictions and actual experiment observations. The integral part of the B2B-DC framework is approximation of the $M(x)$ outputs for given QoI by quadratic surrogate models ${ }^{7}$, and hence the feasible set, can be define as

$$
\Phi:=\left\{x \in \mathrm{H}: L_{e} \leq M_{e}(x) \leq U_{e} \quad \forall \mathrm{e}\right\}
$$

where $M_{e}(x)$ designates a surrogate model of $e$-th QoI. A parameter value that is not in $\Phi$ is at odds with at least one of these constraints.

In this way, the first "bound" in the "bound-to-bound" nomenclature is associated with (a) the form of the prior information, namely that the true model parameters must be both contained in the parameter hypercube $\mathrm{H}$ (in the form of bounds on the components), and (b) the true parameters must result in model predictions of all training experiments that are within the measurement bounds declared by the experimenters, namely $L_{e} \leq M_{e}(x) \leq U_{e}$ for all $e$. Together, these are the "bounds" that define $\Phi$. The following B2B-DC computations (model parameter analysis and optimization) can be performed only if the feasible set $\Phi$ is non-empty. A parameter value that is not in $\boldsymbol{\Phi}$ is at odds with at least one of these constraints.

Dataset consistency is analysis that examines the existence of a feasible parameter vector by determining the consistency measure ${ }^{2} C_{\mathrm{D}}$ of dataset $\mathbf{D}$,

$$
\begin{aligned}
C_{\mathrm{D}}=\max _{\gamma, x \in \mathrm{F}} \gamma, \text { subject to (for all } e \text { ): } \\
\quad(1-\gamma) \frac{L_{e}-U_{e}}{2} \leq M_{e}(x)-\frac{U_{e}+L_{e}}{2} \\
M_{e}(x)-\frac{U_{e}+L_{e}}{2} \leq(1-\gamma) \frac{U_{e}-L_{e}}{2} .
\end{aligned}
$$

In this definition, the original constraints (1) are augmented with a scalar $\gamma$, where positive values of $\gamma$ imply tightening of the constraint (dataset is consistent) and negative values imply loosening (dataset is inconsistent). The consistency measure, $C_{\mathrm{D}}$, quantifies how much the constraints can be tightened while still ensuring the existence of a set of parameter values whose associated model predictions match (within the bounds) the experimental QoI.

Model prediction is the prediction interval for property $\mathrm{P}$ by model $M_{\mathrm{P}}$ that is consistent with all of the model/observation pairs in the dataset. The B2B-DC computation expresses that into two optimization problems for the lower and upper interval endpoints, $L_{\mathrm{P}}$ and $U_{\mathrm{P}}$,

$$
\begin{aligned}
L_{\mathrm{P}} & :=\min _{x \in \mathrm{F}} M_{\mathrm{P}}(x) \\
U_{\mathrm{P}} & :=\max _{x \in \mathrm{F}} M_{\mathrm{P}}(x)
\end{aligned}
$$

The length $U_{\mathrm{P}}-L_{\mathrm{P}}$ quantifies the amount of uncertainty in $M_{\mathrm{P}}$ 's value conditioned on the fact that the true parameter vector is contained in the feasible set $\boldsymbol{\Phi}$.

The results of the proposed analysis suggest a sequential procedure with step-by-step identification of outliers and inspection of the causes. The analysis identifies a specific direction to follow for improving dataset consistency and provides an estimate of the extent of possible improvement. Altogether, this numerical approach offers a tool for assessing experimental observations and model building and improvement. 
In the present paper Data Collaboration module of PrIme ${ }^{1}$ was applied to the $\mathrm{H}_{2} / \mathrm{CO}$ sub-system of the kinetic model $^{9}$ to

1) test the numerical algorithms, modules and user interface of the PrIMe;

2) investigate an algorithm of the PrIMe dataset construction;

3) test the different optimization strategies of chemical kinetic model.

The $\mathrm{CO} / \mathrm{H}_{2}$ mixture oxidation chemistry is the principal building block in the hierarchy of hydrocarbon oxidation models. The main part of the most important reactions influencing the combustion of different types of hydrocarbons follows from this reaction sub-system. In recent years, the role of syngas in sustainable combustion processes and promising syngas utilization for power generation triggered further characterization of the $\mathrm{CO} / \mathrm{H}_{2}$ combustion system. As a result, extensive experimental and numerical studies ${ }^{10}$ have been performed to investigate the $\mathrm{CO} / \mathrm{H}_{2}$ oxidation mechanism comprehensively.

\section{PrIMe DataSet}

\section{A. Reaction Model}

The $\mathrm{H}_{2} / \mathrm{CO}$ sub-model (6 elements, 17 species, 73 reactions) of $\mathrm{C}_{1}-\mathrm{C}_{2}$ reaction mechanism ${ }^{9}$ was used to perform systematic uncertainty and consistency analyses with the Data Collaboration module of PrIme to obtain the feasible set sampling for the base $\mathrm{H}_{2} / \mathrm{CO}$ chemistry of DLR reaction data base. The reaction rate coefficients in the examined sub-model were reviewed with further attention to the pressure depending and multichannel reactions. In comparison to the study, ${ }^{9}$ the reaction rate coefficients for $\mathrm{OH}+\mathrm{OH}(+\mathrm{M})=\mathrm{H}_{2} \mathrm{O}_{2}(+\mathrm{M})$ and $\mathrm{CO}+\mathrm{O}(+\mathrm{M})=\mathrm{CO} 2(+\mathrm{M})$ were replaced with values following from. ${ }^{11-13}$ The input model together with results of validation can be found online: https://teamsites-extranet.dlr.de/vt/DLR-Mechanism/SitePages/Home.aspx. The uncertainty factors for rate coefficients were assumed equal to the proposed ones in the sources or evaluated from statistical treatment of the different data:

$$
\begin{aligned}
& f_{u}(T)=\frac{k_{\text {upper }}(T)}{k_{0}(T)} \\
& f_{l}(T)=\frac{k_{0}(T)}{k_{\text {low }}(T)},
\end{aligned}
$$

where $k_{0}$ is the nominal rate coefficient, $k_{\text {low }}$ and $k_{\text {upper }}$ are lower and upper bounds.

The studied sub-model was extended with $\mathrm{OH}^{*}$ reaction sub-mechanism from ${ }^{14}$ to reproduce more precisely the ignition delay times recorded in shock tubes by the $\mathrm{OH}^{*}$ chemiluminescent measurements and was presented in the xml format adopted in PrIMe. ${ }^{1}$ A preferred key (or PrIMe ID) was prescribed to each structural element in the reaction scheme. Each structural element has a link with the reference information file. Such constructed set of files defines the reaction model $M(x)$ recorded in PrIMe. The model active parameters, i.e., pre-exponential factors of the reaction rate coefficients of the most influential reactions, used for the feasible set construction were identified via sensitivity analysis performed for each QoI. They are reported in Table1.

\section{B. Ignition-delay-time QoI}

Quantification of uncertainties in the shock tube is ultimately needed prior to undertaking any tuning of the kinetic parameters to match ignition targets. If some active phenomena in the shock tube experiments cannot be described by assuming homogeneous conditions (constant V, U system) behind the reflected shock, they are classified as "non-idealities" in the shock tube experiments. ${ }^{22-31}$ Both, facility-dependent effects and energy-release phenomena can increase the non-idealities and influence the instrument readings, thus adding to the uncertainty of experimental data. For the syngas mixtures, the two regimes of ignition should be recognized: weak ignition - the non-uniform and distributed ignition and strong ignition- initiated by auto ignition at the end wall of the shock-tube and propagating through the mixture. ${ }^{27}$ 
Although, the non-idealities present in shock tubes have been well-discussed, ${ }^{22-31}$ the quantitative evaluation of their effects on the reported ignition delay data is a very crucial problem. To evaluate the uncertainty bounds of the measured observations included in the dataset, the empirical algorithm is proposed. For that, the most strong non-

Table1. Active variables

\begin{tabular}{|c|c|c|c|c|c|c|c|}
\hline$\#$ & active variables & $A$ & $n$ & $E_{\mathrm{a}}(\mathrm{K})$ & $\mathrm{Ub}$ & $\mathrm{Lb}$ & Ref. \\
\hline (R1) & $\mathrm{H}_{2}+\mathrm{O}_{2}<=>\mathrm{OH}+\mathrm{OH}$ & $2.400 \mathrm{E}+13$ & 0.4700 & 35121.00 & 0.1 & 10 & 50 \\
\hline (R2) & $\mathrm{H}+\mathrm{HO}_{2}<=>\mathrm{O}_{2}+\mathrm{H}_{2}$ & $2.000 \mathrm{E}+14$ & 0.0000 & 1030.00 & 0.50 & 2.00 & $15 * 2$ \\
\hline (R3) & $\mathrm{H}+\mathrm{O}_{2}(+\mathrm{M})<=>\mathrm{HO}_{2}(+\mathrm{M})$ & $4.660 \mathrm{E}+12$ & 0.4400 & 0.00 & 0.85 & 1.15 & 16 \\
\hline (R4) & $\mathrm{H}+\mathrm{H}+\mathrm{M}<=>\mathrm{H}_{2}+\mathrm{M}$ & $7.470 \mathrm{E}+17$ & -1.0000 & 0.00 & 0.32 & 3.16 & 17 \\
\hline (R5) & $\mathrm{CO}+\mathrm{O}_{2}<=>\mathrm{CO}_{2}+\mathrm{O}$ & $1.260 \mathrm{E}+13$ & 0.0000 & 23682.94 & 0.20 & 5.01 & 18 \\
\hline (R6) & $\mathrm{H}+\mathrm{HCO}<=>\mathrm{H}_{2}+\mathrm{CO}$ & $9.000 \mathrm{E}+13$ & 0.0000 & 0.00 & 0.50 & 2.00 & 15 \\
\hline (R7) & $\mathrm{HCO}+\mathrm{O}_{2}<=>\mathrm{CO}+\mathrm{HO}_{2}$ & $1.350 \mathrm{E}+10$ & 0.6800 & -236.00 & 0.40 & 2.50 & 15 \\
\hline (R8) & $\mathrm{CO}+\mathrm{HO}_{2}<=>\mathrm{CO}_{2}+\mathrm{OH}$ & $1.150 \mathrm{E}+05$ & 2.2800 & 8775.00 & 0.32 & 3.16 & 19 \\
\hline (R9) & $\mathrm{CO}+\mathrm{O}(+\mathrm{M})<=>\mathrm{CO}_{2}(+\mathrm{M})$ & $1.362 \mathrm{E}+10$ & 0.0000 & 1242.0 & 0.32 & 3.16 & 20 \\
\hline (R10) & $\mathrm{H}+\mathrm{O}+\mathrm{M}<=>\mathrm{OH}+\mathrm{M}$ & $7.730 \mathrm{E}+18$ & -1.0000 & 0.00 & 0.20 & 5.01 & 18 \\
\hline$(\mathrm{R} 11)$ & $\mathrm{H}_{2}+\mathrm{O}<=>\mathrm{OH}+\mathrm{H}$ & $3.820 \mathrm{E}+12$ & 0.0000 & 4000.00 & 0.63 & 1.58 & 15 \\
\hline (R12) & $\mathrm{H}+\mathrm{HO}_{2}<=>\mathrm{O}+\mathrm{H}_{2} \mathrm{O}$ & $1.440 \mathrm{E}+12$ & 0.0000 & 0.00 & 0.32 & 3.16 & 15 \\
\hline (R13) & $\mathrm{H}+\mathrm{H}_{2} \mathrm{O}_{2}<=>\mathrm{OH}+\mathrm{H}_{2} \mathrm{O}$ & $1.020 \mathrm{E}+13$ & 0.0000 & 1800.58 & 0.50 & 2.00 & 15 \\
\hline (R14) & $\mathrm{OH}+\mathrm{OH}<=>\mathrm{O}+\mathrm{H}_{2} \mathrm{O}$ & $3.350 \mathrm{E}+04$ & 2.4200 & -970.00 & 0.70 & 1.40 & 15 \\
\hline (R15) & $\mathrm{O}+\mathrm{H}_{2} \mathrm{O}_{2}<=>\mathrm{OH}+\mathrm{HO}_{2}$ & $8.430 \mathrm{E}+11$ & 0.0000 & 2000.00 & 0.50 & 2.00 & 15 \\
\hline (R16) & $\mathrm{H}+\mathrm{HO}_{2}<=>\mathrm{OH}+\mathrm{OH}$ & $4.000 \mathrm{E}+14$ & 0.0000 & 700.00 & 0.70 & 1.40 & 15 \\
\hline (R17) & $\mathrm{OH}+\mathrm{HO}_{2}<=>\mathrm{O}_{2}+\mathrm{H}_{2} \mathrm{O}$ & $2.890 \mathrm{E}+13$ & 0.0000 & -250.00 & 0.63 & 1.60 & 15 \\
\hline (R18) & $\mathrm{O}+\mathrm{HO}_{2}<=>\mathrm{OH}+\mathrm{O}_{2}$ & $1.630 \mathrm{E}+13$ & 0.0000 & -224.00 & 0.32 & 3.16 & 15 \\
\hline (R19) & $\mathrm{OH}+\mathrm{H}_{2}<=>\mathrm{H}+\mathrm{H}_{2} \mathrm{O}$ & $2.160 \mathrm{E}+08$ & 1.5200 & 1740.00 & 0.65 & 1.63 & 15 \\
\hline (R20) & $\mathrm{H}+\mathrm{O}_{2}<=>\mathrm{O}+\mathrm{OH}$ & $1.900 \mathrm{E}+14$ & -0.0970 & 7560.00 & 0.80 & 1.26 & 15 \\
\hline (R21) & $\mathrm{H}+\mathrm{H}_{2} \mathrm{O}_{2}<=>\mathrm{HO}_{2}+\mathrm{H}_{2}$ & $1.690 \mathrm{E}+12$ & 0.0000 & 1889.58 & 0.32 & 3.16 & 15 \\
\hline (R22) & $\mathrm{OH}+\mathrm{H}_{2} \mathrm{O}_{2}<=>\mathrm{HO}_{2}+\mathrm{H}_{2} \mathrm{O}$ & $1.930 \mathrm{E}+12$ & 0.0000 & 215.00 & 0.50 & 2.00 & 15 \\
\hline (R23) & $\mathrm{HO}_{2}+\mathrm{HO}_{2}<=>\mathrm{O}_{2}+\mathrm{H}_{2} \mathrm{O}_{2}$ & $1.320 \mathrm{E}+11$ & 0.0000 & -820.30 & 0.40 & 2.50 & 15 \\
\hline (R24) & $\mathrm{O}+\mathrm{HCO}<=>\mathrm{OH}+\mathrm{CO}$ & $3.010 \mathrm{E}+13$ & 0.0000 & 0.00 & 0.50 & 2.00 & 15 \\
\hline (R25) & $\mathrm{O}+\mathrm{HCO}<=>\mathrm{H}+\mathrm{CO}_{2}$ & $3.010 \mathrm{E}+13$ & 0.0000 & 0.00 & 0.50 & 2.00 & 15 \\
\hline $\mathrm{R}(26)$ & $\mathrm{CO}+\mathrm{OH}<=>\mathrm{CO}_{2}+\mathrm{H}$ & $1.010 \mathrm{E}+13$ & 0.0000 & 8050.00 & 0.80 & 1.26 & 15 \\
\hline $\mathrm{R}(27)$ & $\mathrm{HCO}+\mathrm{O}_{2}<=>\mathrm{OH}+\mathrm{CO}_{2}$ & $1.350 \mathrm{E}+10$ & 0.6800 & -236.00 & 0.40 & 2.50 & 15 \\
\hline $\mathrm{R}(28)$ & $\mathrm{HCO}+\mathrm{M}<=>\mathrm{CO}+\mathrm{H}+\mathrm{M}$ & $4.750 \mathrm{E}+11$ & 0.6600 & 7485.00 & 0.50 & 2.00 & 21 \\
\hline
\end{tabular}

ideality phenomena ${ }^{22-31}$ were determined across the investigations and the facility-related and fuel-related factors, which affect these phenomena, have been identified.

The dominant non-ideality phenomena were attributed to two gas dynamics effects: i) boundary layer formation after incident shock wave interacts with reflected shock-wave (resulting in inhomogeneities of $T$ and $p$ behind the shock-wave and shock bifurcations); ii) post-shock compression (interaction of the reflected shock-wave with the contact surface). The second most important phenomena influencing the measurements uncertainty is energyrelease: the weak regime (the non-uniform/distributed ignition) and the strong regime (initiated by auto ignition at the end wall of the shock-tube) of ignition. The factors which influence these phenomena are: operating conditions; driven section length; driven section diameter; measurement duration; mixture dilution and nature of Carrier Gas (CG). 
In the first column of the Table 2, factors, which influence the shock tube measurement error, are summarized. In the second column operating conditions which influence these factors are indicated. The possible errors, caused by these factors and the parameter change, leading to possible error increase, are evaluated in the third column.

It was found that experimental data obtained by using large diameter shock tubes $(\sim 10 \mathrm{~cm})$, dilute fuel/oxidizer mixtures in monoatomic gases, and short test times (less than about $500 \mu \mathrm{s}$ ) have the lowest uncertainty level. A correspondence with the diameter of the shock-tube and weak ignition is found: the larger diameter leading to an ignition delay close to that of a homogeneous reactor.

Table 2.Experimental Ignition Delay Data: uncertainty factors

\begin{tabular}{|c|c|c|}
\hline $\begin{array}{l}\text { Facility-related and fuel-related } \\
\text { factors affecting the non-ideality } \\
\text { phenomena and uncertainty in } \\
\text { shock tube measurements }\end{array}$ & $\begin{array}{l}\text { Operating conditions } \\
\text { influencing the factors }\end{array}$ & $\begin{array}{l}\text { Contribution in } \\
\text { uncertainty } \\
\text { correlated with } \\
\text { Operating } \\
\text { conditions }\end{array}$ \\
\hline Weak and strong ignition (fuel) & $\mathrm{T}, \mathrm{p}, \phi, \mathrm{CG}$ & $\begin{array}{l}\text { Low } \mathrm{T}, \mathrm{p}, \phi>0.3 \\
\sim 10 \text { times }\end{array}$ \\
\hline $\begin{array}{l}\text { Non-ideal gas dynamics behind } \\
\text { the reflected shock wave ( } T, p \\
\text { non-uniformities) }\end{array}$ & $\mathrm{T}, \mathrm{p}, \mathrm{t}_{\text {meas }}, \mathrm{CG}$ & $\begin{array}{l}27 \%-17 \% \\
\text { Small Dilution } \downarrow\end{array}$ \\
\hline Post-shock compression & $\mathrm{T}, \mathrm{p}, \mathrm{t}_{\text {meas }}, \mathrm{CG}$ & $\begin{array}{l}\mathrm{dP} / \mathrm{dT} \approx 2-6 \% / \mathrm{ms} \\
\mathrm{dT} / \mathrm{dt} \approx 1.2 \% / \mathrm{ms}\end{array}$ \\
\hline Radical impurities & T, p, CG, Person & dilution $\uparrow, \mathrm{T}, \mathrm{p} \downarrow ;$ ? \\
\hline Temperature measurements & Measurement location & $5 \%$ \\
\hline Pressure measurements & $\begin{array}{l}\mathrm{T} \text {, Measurement } \\
\text { location }\end{array}$ & $10 \%$ \\
\hline $\begin{array}{l}\text { Concentration measurements } \\
\text { (the steepest rate of change) }\end{array}$ & $\begin{array}{l}\mathrm{T}, \text { Measurement } \\
\text { location }\end{array}$ & $5 \%$ \\
\hline
\end{tabular}

Table 3. Ignition delay time measurements selected model validation.

\begin{tabular}{|c|c|c|c|c|}
\hline $\boldsymbol{P}$, MPa & Composition & $\varphi$ & $\boldsymbol{T}_{5}, \mathbf{K}$ & Ref. \\
\hline $0.061-1.82$ & $20 \% \mathrm{CO} / 80 \% \mathrm{H}_{2}$ & 0.5 & $890-1285$ & Kalitan et al. $^{32}$ \\
& $40 \% \mathrm{CO} / 60 \% \mathrm{H}_{2}$ & & & \\
& $80 \% \mathrm{CO} / 20 \% \mathrm{H}_{2}$ & & & \\
& $90 \% \mathrm{CO} / 10 \% \mathrm{H}_{2}$ & & & \\
\hline $1.11-3.24$ & $\mathrm{CO} / \mathrm{H}_{2} / \mathrm{CO}_{2} / \mathrm{O}_{2} / \mathrm{N}_{2}$ & 0.5 & $630-1150$ & Petersen et al. $^{33}$ \\
\hline $0.12-0.14$ & $80 \% \mathrm{CO} / 20 \% \mathrm{H}_{2}$ & 0.5 and & $909-965$ & Mertens et al. $^{34}$ \\
& $90 \% \mathrm{CO} / 10 \% \mathrm{H}_{2}$ & 1.0 & & \\
\hline $1.41-1.72$ & $50 \% \mathrm{CO} / 50 \% \mathrm{H}_{2}$ & $0.5-1.0$ & $1048-1259$ & Herzler et al $^{35}$ \\
& $95 \% \mathrm{CO} / 5 \% \mathrm{H}_{2}$ & & & \\
\hline $0.1 ; 0.61 ; 1.21 ;$ & Dilution $1: 2,5,10$ & & & Krejci et al. $^{36}$ \\
& $50 \% \mathrm{CO} / 50 \% \mathrm{H}_{2}$ & 0.5 & $980-2004$ & \\
\hline 3.24 & $90 \% \mathrm{CO} / 510 \% \mathrm{H}_{2}$ & & & \\
& Dilution $98 \% \mathrm{Ar}$ & & & \\
\hline
\end{tabular}

It was assumed, that in the best case (strong ignition, diluted mixture, $t_{\text {meas }}=50 \mathrm{~ms}-500 \mathrm{~ms}$, shock tube diameter $>10 \mathrm{~cm}$, length of driven-section $>8 \mathrm{~m}$ ) the uncertainty can be assumed $\sim 15 \%$. Deviations from these conditions are evaluated by adding a $5 \%$ uncertainty for each criterion not satisfied to the ideal case. For measured 
ignition delay time longer as $1000 \mu \mathrm{s} 5 \%$ uncertainty is added per every $1000 \mu$ s. Radical impurities were evaluated as extra $5 \%$ uncertainty to the ideal case.

Table 4. Ignition delay QoI selected for the analysis.

\begin{tabular}{|c|c|c|c|c|c|c|c|c|}
\hline Ref. & $\begin{array}{c}\text { Driven } \\
\text { section } \\
\text { length, } \\
\text { m }\end{array}$ & $\begin{array}{c}\text { Internal } \\
\text { diameter, } \\
\mathbf{c m}\end{array}$ & $\begin{array}{c}\text { Temperature } \\
\text { interval, } \mathrm{K}\end{array}$ & $\begin{array}{c}\text { Pressure, } \\
\text { MPa }\end{array}$ & $\varphi$ & Dilution & $t_{\text {meas },} \mu \mathrm{s}$ & $\begin{array}{c}\text { Integrated } \\
\text { uncertainty } \\
\%\end{array}$ \\
\hline $\begin{array}{l}\text { Kalitan } \\
\text { et al. }\end{array}$ & 10.7 & 16.2 & \begin{tabular}{|l|}
$<1000$ \\
$+5 \%$ \\
\end{tabular} & $<1.5$ & 0.5 & $\begin{array}{l}\text { none } \\
+\mathbf{5} \% \\
\end{array}$ & $100-500$ & 30 \\
\hline $\begin{array}{l}\text { Kalitan } \\
\text { et al. }\end{array}$ & 10.7 & 16.2 & $\begin{array}{l}<1000 \\
+5 \%\end{array}$ & $<1.5$ & 0.5 & $\begin{array}{l}\text { none } \\
+5 \%\end{array}$ & $\begin{array}{l}500-1000 \\
+5 \%\end{array}$ & 35 \\
\hline $\begin{array}{l}\text { Kalitan } \\
\text { et al. }\end{array}$ & 10.7 & 16.2 & $\begin{array}{l}<1000 \\
+5 \%\end{array}$ & $<1.5$ & 0.5 & $\begin{array}{l}\text { none } \\
+5 \%\end{array}$ & $\begin{array}{l}1000-2000 \\
+10 \%\end{array}$ & 40 \\
\hline $\begin{array}{l}\text { Kalitan } \\
\text { et al. }\end{array}$ & 10.7 & 16.2 & $>1000$ & $<1.5$ & 0.5 & $\begin{array}{l}\text { none } \\
+5 \%\end{array}$ & $\begin{array}{l}100-600 \\
+5 \%\end{array}$ & 30 \\
\hline $\begin{array}{l}\text { Petersen } \\
\text { et al. }\end{array}$ & 10.7 & 16.2 & $\begin{array}{l}<1000 \\
+5 \%\end{array}$ & $>1.5+5 \%$ & 0.5 & $\begin{array}{l}\text { none } \\
+5 \%\end{array}$ & $\begin{array}{l}500-1000 \\
+5 \%\end{array}$ & 40 \\
\hline $\begin{array}{l}\text { Petersen } \\
\text { et al. }\end{array}$ & 10.7 & 16.2 & $\begin{array}{l}<1000 \\
+5 \%\end{array}$ & $>1.5+5 \%$ & 0.5 & $\begin{array}{l}\text { none } \\
+5 \%\end{array}$ & $\begin{array}{l}1000-2000 \\
+10 \%\end{array}$ & 45 \\
\hline $\begin{array}{l}\text { Petersen } \\
\text { et al. }\end{array}$ & 10.7 & 16.2 & $\begin{array}{l}<1000 \\
+5 \%\end{array}$ & $>1.5+5 \%$ & 0.5 & $\begin{array}{l}\text { none } \\
+5 \%\end{array}$ & $\begin{array}{l}2000-3000 \\
+15 \%\end{array}$ & 50 \\
\hline $\begin{array}{l}\text { Mertens } \\
\text { et al. }\end{array}$ & 10.7 & 16.2 & $\begin{array}{l}<1000 \\
+5 \%\end{array}$ & $<1.5$ & $0.5-1$ & $\begin{array}{l}\text { none } \\
+5 \%\end{array}$ & $\begin{array}{l}600-2000 \\
+10 \%\end{array}$ & 40 \\
\hline $\begin{array}{l}\text { Mertens } \\
\text { et al. }{ }^{34}\end{array}$ & 10.7 & 16.2 & $\begin{array}{l}<1000 \\
+5 \%\end{array}$ & $<1.5$ & $0.5-1$ & yes & $\begin{array}{l}600-1200 \\
+10 \%\end{array}$ & 35 \\
\hline $\begin{array}{l}\text { Herzler } \\
\text { et al. }\end{array}$ & 11.12 & 9.82 & $>1000$ & $>1.5+5 \%$ & 0.5 & yes & $300-500$ & 25 \\
\hline $\begin{array}{l}\text { Herzler } \\
\text { et al. }\end{array}$ & 11.12 & 9.82 & $>1000$ & $>1.5+5 \%$ & 0.5 & yes & $\begin{array}{l}500-1000 \\
+5 \%\end{array}$ & 30 \\
\hline $\begin{array}{l}\text { Herzler } \\
\text { et al. }\end{array}$ & 11.12 & 9.82 & $>1000$ & $>1.5+5 \%$ & 0.5 & yes & $\begin{array}{l}500-1000 \\
+5 \%\end{array}$ & 30 \\
\hline $\begin{array}{l}\text { Kéromnès } \\
\text { et al. }^{36}\end{array}$ & $\begin{aligned} & 4.72 \\
+ & 5 \%\end{aligned}$ & 15.24 & $>1000$ & $<1.5$ & $0.5 ; 1$ & yes & $20-500$ & 25 \\
\hline 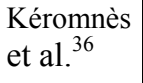 & $\begin{array}{r}4.72 \\
+5 \%\end{array}$ & 15.24 & $>1000$ & $<1.5$ & $0.5 ; 1$ & yes & $\begin{array}{l}500-1000 \\
+5 \%\end{array}$ & 30 \\
\hline $\begin{array}{l}\text { Kéromnès } \\
\text { et al. }\end{array}$ & $\begin{aligned} & 4.72 \\
+ & 5 \%\end{aligned}$ & 15.24 & $>1000$ & $>1.5+5 \%$ & $0.5 ; 1$ & yes & $20-500$ & 30 \\
\hline $\begin{array}{l}\text { Kéromnès } \\
\text { et al. }^{36}\end{array}$ & $\begin{aligned} & 4.72 \\
+ & 5 \%\end{aligned}$ & 15.24 & $>1000$ & $>1.5+5 \%$ & $0.5 ; 1$ & yes & $\begin{array}{l}500-1000 \\
+5 \%\end{array}$ & 35 \\
\hline $\begin{array}{l}\text { Kéromnès } \\
\text { et al. }^{36}\end{array}$ & $\begin{aligned} & 4.72 \\
+ & 5 \%\end{aligned}$ & 15.24 & $>1000$ & $>1.5+5 \%$ & $0.5 ; 1$ & yes & $\begin{array}{l}1000-2000 \\
+10 \%\end{array}$ & 40 \\
\hline
\end{tabular}

6

American Institute of Aeronautics and Astronautics 
In Table 3 the shock tube experiments ${ }^{32-36}$ used for model validation are collected. On this step of the methodology testing, we selected 95 ignition delay targets for the analysis. The results of uncertainty evaluation obtained with the proposed empirical rule for the syngas ignition delay time experimental values ${ }^{32-36}$ to be included in the PrIMe dataset, are collected in Table 4.

Table 5. Evaluation of uncertainty intervals for laminar flame experimental data selected for QoI

\begin{tabular}{|c|l|r|l|r|l|r|}
\hline$\varphi$ & $\boldsymbol{p}, \boldsymbol{M P a}$ & \multicolumn{1}{|c|}{ Error } & $\boldsymbol{p}, \boldsymbol{M P a}$ & \multicolumn{1}{c|}{ Error } & $\boldsymbol{p}, \boldsymbol{M P a}$ & Error \\
\hline $0.5-2$ & $0.1 \div 0.51$ & $10 \%$ & $0.51 \div 1.01$ & $15 \%$ & $>1.01$ & $20 \%$ \\
\hline $2.0 \div 3.0$ & $0.1 \div 0.51$ & $15 \%$ & $0.51 \div 1.01$ & $20 \%$ & $>1.01$ & $25 \%$ \\
\hline$>3.0$ & $0.1 \div 0.51$ & $20 \%$ & $0.51 \div 1.01$ & $25 \%$ & $>1.01$ & $30 \%$ \\
\hline
\end{tabular}

\section{Laminar-flame-velocity QoI}

Syngas flame velocities at 0.1-0.5 MPa have been investigated by using almost all known techniques. ${ }^{37-40}$ The flame velocity data at high pressures are relatively sparse. Experimentalists consider the current uncertainties of laminar flame speed measurements to be in a range of about 5-10\%, but also indicating its increase with pressure $(>0.5 \mathrm{MPa})$ and fuel-air ratio $(\varphi>2){ }^{37-40}$

Uncertainty bounds of experimental data were evaluated from studies ${ }^{37-40}$ and analysis of the current syngas atmospheric laminar flame speed data distribution, which can be found in ${ }^{41}$ From the data analysis following from ${ }^{37-}$ ${ }^{44}$, the uncertainty of available data can be assumed to be $10 \%$ for $\varphi<2,15 \%$ for $2<\varphi<3$, and $20 \%$ for $\varphi>3$.

Table 6. Laminar flame speed measurements selected for model validation

\begin{tabular}{|c|c|c|c|c|c|c|c|}
\hline \# & Ref. & Mixture & $p, M P a$ & Prime ID & To, K & $\varphi$ & Error \\
\hline 1 & Sun et al. ${ }^{19}$ & $50 / 50 \% \mathrm{CO} / \mathrm{H}_{2} /$ air & 0.1 & $\mathrm{a} 00000128$ & 300 & 0.8 & $10 \%$ \\
\hline 2 & Sun et al. ${ }^{19}$ & $50 / 50 \% \mathrm{CO} / \mathrm{H}_{2} /$ air & 0.1 & $\mathrm{a} 00000129$ & 300 & 1.2 & $10 \%$ \\
\hline 3 & Sun et al. ${ }^{19}$ & $50 / 50 \% \mathrm{CO} / \mathrm{H}_{2} /$ air & 0.1 & $\mathrm{a} 00000130$ & 300 & 2.5 & $15 \%$ \\
\hline 4 & Sun et al. ${ }^{19}$ & $95 / 5 \% \mathrm{CO} / \mathrm{H}_{2} / \mathrm{He}$ & 0.5 & $\mathrm{a} 00000249$ & 300 & 2 & $15 \%$ \\
\hline 5 & Sun et al. ${ }^{19}$ & $95 / 5 \% \mathrm{CO} / \mathrm{H}_{2} / \mathrm{He}$ & 1.0 & $\mathrm{a} 00000250$ & 300 & 0.75 & $15 \%$ \\
\hline 6 & Sun et al. ${ }^{19}$ & $95 / 5 \% \mathrm{CO} / \mathrm{H}_{2} / \mathrm{He}$ & 1.0 & $\mathrm{a} 00000252$ & 300 & 1.4 & $15 \%$ \\
\hline 7 & Sun et al. ${ }^{19}$ & $95 / 5 \% \mathrm{CO} / \mathrm{H}_{2} / \mathrm{He}$ & 2.0 & $\mathrm{a} 00000253$ & 300 & 1 & $20 \%$ \\
\hline 8 & Sun et al. ${ }^{19}$ & $95 / 5 \% \mathrm{CO} / \mathrm{H}_{2} / \mathrm{He}$ & 4.0 & $\mathrm{a} 00000257$ & 300 & 1.4 & $20 \%$ \\
\hline 9 & Sun et al. ${ }^{19}$ & $50 / 50 \% \mathrm{CO} / \mathrm{H}_{2} / \mathrm{He}$ & 0.5 & $\mathrm{a} 00000124$ & 300 & 3.5 & $25 \%$ \\
\hline 10 & Sun et al. ${ }^{19}$ & $50 / 50 \% \mathrm{CO} / \mathrm{H}_{2} / \mathrm{He}$ & 1.0 & $\mathrm{a} 00000125$ & 300 & 1 & $15 \%$ \\
\hline 11 & Sun et al. ${ }^{19}$ & $50 / 50 \% \mathrm{CO} / \mathrm{H}_{2} / \mathrm{He}$ & 1.0 & $\mathrm{a} 00000126$ & 300 & 1.8 & $15 \%$ \\
\hline 12 & Sun et al. ${ }^{19}$ & $50 / 50 \% \mathrm{CO} / \mathrm{H}_{2} / \mathrm{He}$ & 1.0 & $\mathrm{a} 00000127$ & 300 & 3.5 & $20 \%$ \\
\hline 13 & Natarajan et al. $^{42}$ & $50 / 50 \% \mathrm{CO} / \mathrm{H}_{2} /$ air & 0.1 & $\mathrm{a} 00000282$ & 700 & 0.73 & $10 \%$ \\
\hline 14 & Natarajan et al. $^{42}$ & $50 / 50 \% \mathrm{CO} / \mathrm{H}_{2} /$ air & 0.1 & $\mathrm{a} 00000280$ & 700 & 0.9 & $10 \%$ \\
\hline 15 & Sun et al. ${ }^{19}$ & $95 / 5 \% \mathrm{CO} / \mathrm{H}_{2} /$ air & 0.1 & $\mathrm{a} 00000260$ & 300 & 1 & $10 \%$ \\
\hline 16 & Sun et al. ${ }^{19}$ & $95 / 5 \% \mathrm{CO} / \mathrm{H}_{2} /$ air & 0.1 & $\mathrm{a} 00000261$ & 300 & 1.5 & $10 \%$ \\
\hline 17 & Hassan et al. $^{43}$ & $95 / 5 \% \mathrm{CO} / \mathrm{H}_{2} /$ air & 0.05 & $\mathrm{a} 00000269$ & 300 & 1 & $10 \%$ \\
\hline 18 & Hassan et al. $^{43}$ & $95 / 5 \% \mathrm{CO} / \mathrm{H}_{2} /$ air & 0.1 & $\mathrm{a} 00000271$ & 300 & 0.6 & $10 \%$ \\
\hline 19 & Sun et al. ${ }^{19}$ & $95 / 5 \% \mathrm{CO} / \mathrm{H}_{2} / \mathrm{He}$ & 2.0 & $\mathrm{x} 00000460$ & 300 & 1.6 & $20 \%$ \\
\hline 20 & Sun et al. ${ }^{19}$ & $95 / 5 \% \mathrm{CO} / \mathrm{H}_{2} / \mathrm{He}$ & 2.0 & $\mathrm{x} 00000460$ & 300 & 3 & $25 \%$ \\
\hline 21 & Sun et al. ${ }^{19}$ & $95 / 5 \% \mathrm{CO} / \mathrm{H}_{2} / \mathrm{He}$ & 4.0 & x00000461 & 300 & 2 & $25 \%$ \\
\hline 22 & Natarajan et al. ${ }^{44}$ & $50 / 50 \% \mathrm{CO} / \mathrm{H}_{2} / \mathrm{He}$ & 1.5 & x00000471 & 600 & 0.6 & $20 \%$ \\
\hline 23 & Natarajan et al. ${ }^{44}$ & $50 / 50 \% \mathrm{CO} / \mathrm{H}_{2} / \mathrm{He}$ & 1.5 & $\mathrm{x} 00000471$ & 600 & 0.6 & $20 \%$ \\
\hline
\end{tabular}


The uncertainties for experimental data measured at higher pressure have been evaluated by adding 5\%. The empirical rule applied for uncertainties evaluation of laminar speed data can be found in Table 5 .

The 23 laminar flame speed data included in the dataset are taken from studies ${ }^{19,42-44}$, Table 6 . They are selected to cover as optimal as possible the full range of operating conditions available in the literature.

A preferred key (or PrIMe ID) was prescribed to each experimental target. In this way, each structural element has a "pointer" to the referenced information and/or file. All the experimental data were documented in the PrIMe Data Warehouse. ${ }^{1}$ Selected for analysis experimental QoI are described in the dataAttribute files of the PrIMe data collection. ${ }^{1}$ These QoI together with the corresponding model $\mathrm{M}_{\mathrm{e}}(\mathrm{x})$ and the experimental and parameter bounds form a dataset. The complete model and experimental data are available in the PrIMe Data Warehouse. ${ }^{1}$

\section{General Results}

The ignition delay times and laminar flame speeds were modeled with numerical tools of PrIMe, ${ }^{1}$ numerical packages CHEMKIN II $^{45}$ and Chemical Workbench. ${ }^{46}$ The ignition delay time was computationally defined by the peak in the $\mathrm{OH}$ or $\mathrm{OH}^{*}$ concentration, temperature, or pressure. It is pointed in the attribute files of PrIMe Warehouse. The thermal diffusion model was applied for calculation of one-dimensional freely propagating laminar premixed flame using CHEMKIN II with over 1000 grid points for each condition.

\section{A. Dataset Consistency (Data Quality)}

The consistency analysis was performed for the dataset that included first 95 QoI of ignition delay times, 23 QoI of laminar flame speed and 28 active parameters (Tables 1, 5, and 6). Initially, before performing the consistency test, 12 experimental QoI were excluded from the dataset because the ignition delay times could not be reproduced at all: the calculated $\mathrm{OH}^{*}$ profile in these cases did not exhibit a maximum. All other 83 ignition delay targets were fitted with quadratic surrogate models with on-design errors not exciding $1 \%$ and off-design errors below or about $2 \%$. The initial results of the consistency analysis indicated a high degree of inconsistency of ignition delay QoI. To bring the dataset to consistency, QoI bounds were changed, as shown in Fig. 1 and in details in Table 6.

The further consistency analysis performed for 83 ignition delay targets and 23 laminar flame speeds determined that ignition-delay QoI with large bound changes, Table 6, and eight computed flame QoI values (flames F2, F4, F13-17, F22 in Table 5) fell outside their respective uncertainty bounds, should be assumed as self-inconsistent data and hence were excluded from the dataset. Self-inconsistency means that no point in the rate constant domain $\boldsymbol{H}$ can reproduce the experimental QoI within its uncertainty bounds.

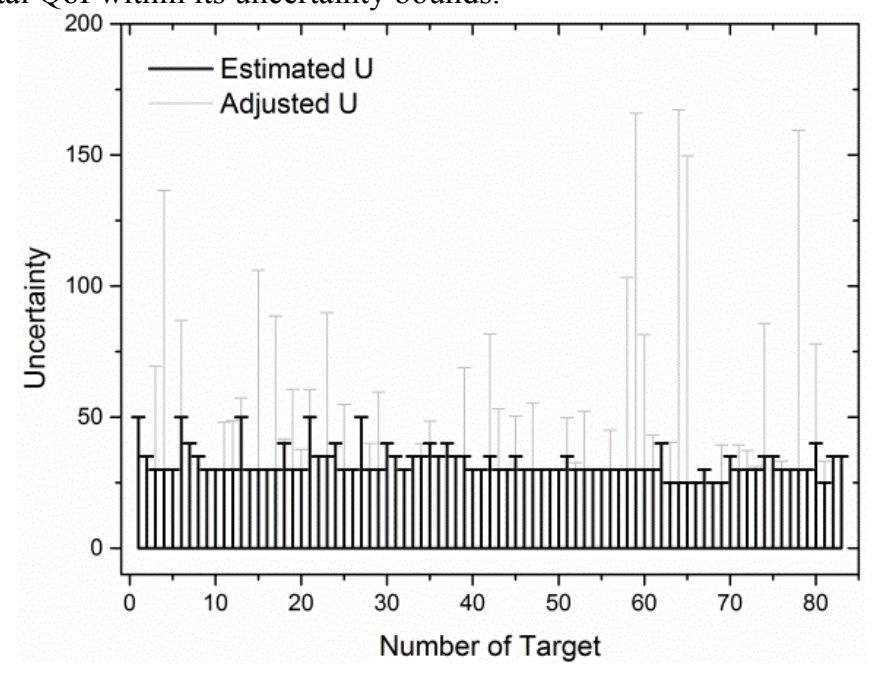

Figure 1. The bound change in ignition delay QoI obtained by data consistency analysis. 
Table 6. Results of B2B-DC analysis for ignition delay time targets (Low T: T<1000K High p:p > 1 MPa). Blue color: targets excluded since calculated profile did not exhibit maximum OH*. Gray color: targets excluded due large UQ

\begin{tabular}{|c|c|c|c|c|c|c|c|}
\hline \# & $\mathbf{T}_{5}, \mathbf{K}$ & $\mathrm{p}_{5}, \mathrm{MPa}$ & Prime ID & $\begin{array}{l}\text { Current } \\
\text { Uncertainty, \% }\end{array}$ & $\begin{array}{l}\text { Minimum } \\
\text { UQ, \% }\end{array}$ & Refs. & Comment \\
\hline 1 & 916 & 0.11 & a00000179 & 50 & & 25 & \\
\hline 2 & 954 & 0.12 & $\mathrm{a} 00000181$ & 35 & & 25 & \\
\hline 3 & 993 & 0.10 & $\mathrm{a} 00000183$ & 30 & 69,4476 & 25 & Low T \\
\hline 4 & 1074 & 0.11 & $\mathrm{a} 00000186$ & 30 & 136,4403 & 25 & \\
\hline 5 & 1151 & 0.1 & $\mathrm{a} 00000188$ & 30 & & 25 & \\
\hline 6 & 914 & 0.11 & $\mathrm{a} 00000105$ & 50 & 86,922 & 25 & Low T \\
\hline 7 & 951 & 0.11 & $\mathrm{a} 00000131$ & 40 & & 25 & \\
\hline 8 & 996 & 0.11 & $\mathrm{a} 00000132$ & 35 & & 25 & \\
\hline 9 & 1072 & 0.11 & $\mathrm{a} 00000133$ & 30 & & 25 & \\
\hline 10 & 1175 & 0.1 & $\mathrm{a} 00000135$ & 30 & & 25 & \\
\hline 11 & 1187 & 0.1 & a00000136 & 30 & 47,986 & 25 & \\
\hline 12 & 1241 & 0.1 & $\mathrm{a} 00000107$ & 30 & 48,6134 & 25 & \\
\hline 13 & 900 & 0.06 & $\mathrm{a} 00000110$ & 50 & 57,3177 & 25 & Low T \\
\hline 14 & 1026 & 0.11 & $\mathrm{a} 00000111$ & 30 & & 25 & \\
\hline 15 & 1162 & 0.1 & $\mathrm{a} 00000112$ & 30 & 105,9988 & 25 & High P \\
\hline 16 & 936 & 0.12 & $\mathrm{a} 00000113$ & 40 & & 25 & \\
\hline 17 & 1015 & 0.11 & $\mathrm{a} 00000189$ & 30 & & 25 & \\
\hline 18 & 1183 & 0.11 & a00000190 & 30 & 88,6136 & 25 & \\
\hline 19 & 929 & 0.26 & $\mathrm{a} 00000191$ & 50 & & 25 & \\
\hline 20 & 992 & 0.26 & $\mathrm{a} 00000192$ & 40 & 41,6159 & 25 & \\
\hline 21 & 1058 & 0.26 & $\mathrm{a} 00000114$ & 30 & 60,6507 & 25 & \\
\hline 22 & 1063 & 0.31 & $\mathrm{a} 00000193$ & 30 & 37,6735 & 25 & \\
\hline 23 & 1015 & 1.39 & $\mathrm{a} 00000213$ & 50 & 60,4698 & 25 & High P \\
\hline 24 & 1114 & 1.51 & a00000194 & 35 & & 25 & \\
\hline 25 & 1190 & 1.70 & $\mathrm{a} 00000115$ & 35 & 89,866 & 25 & High P \\
\hline 26 & 960 & 0.12 & $\mathrm{a} 00000116$ & 40 & & 25 & \\
\hline 27 & 1052 & 0.11 & $\mathrm{a} 00000195$ & 30 & 54,8933 & 25 & \\
\hline 28 & 1197 & 0.11 & $\mathrm{a} 00000196$ & 30 & & 25 & \\
\hline 29 & 981 & 0.27 & a00000197 & 50 & & 25 & \\
\hline 30 & 1048 & 0.25 & $\mathrm{a} 00000198$ & 30 & 40,0207 & 25 & \\
\hline 31 & 1118 & 0.25 & $\mathrm{a} 00000117$ & 30 & 59,5535 & 25 & \\
\hline 32 & 1063 & 1.45 & $\mathrm{a} 00000199$ & 40 & & 25 & \\
\hline 33 & 1126 & 1.20 & a00000200 & 35 & & 25 & \\
\hline 34 & 1265 & 1.73 & a00000118 & 30 & 30,0166 & 25 & \\
\hline
\end{tabular}

9

American Institute of Aeronautics and Astronautics 


\begin{tabular}{|c|c|c|c|c|c|c|c|}
\hline 35 & 968 & 0.12 & $\mathrm{a} 00000307$ & 40 & & 25 & \\
\hline 36 & 1033 & 2.40 & $\mathrm{a} 00000317$ & 35 & & 26 & \\
\hline 37 & 1148 & 2.17 & $\mathrm{a} 00000318$ & 35 & 39,9265 & 26 & High P \\
\hline 38 & 909 & 0.119 & $\mathrm{a} 00000322$ & 40 & & 27 & \\
\hline 39 & 933 & 0.116 & $\mathrm{a} 00000323$ & 40 & 48,481 & 27 & Low T \\
\hline 40 & 947 & 0.121 & $\mathrm{a} 00000324$ & 35 & & 27 & \\
\hline 41 & 932 & 0.14 & $\mathrm{a} 00000223$ & 40 & & 27 & \\
\hline 42 & 956 & 0.14 & $\mathrm{a} 00000224$ & 35 & & 27 & \\
\hline 43 & 965 & 0.15 & $\mathrm{a} 00000225$ & 35 & 68,8672 & 27 & Low T \\
\hline 44 & 1046 & 1.70 & $\mathrm{a} 00000226$ & 30 & & 28 & \\
\hline 45 & 1072 & 1.60 & $\mathrm{a} 00000227$ & 30 & & 28 & \\
\hline 46 & 1132 & 1.64 & $\mathrm{a} 00000228$ & 35 & 81,7297 & 28 & High P \\
\hline 47 & 1107 & 1.64 & $\mathrm{a} 00000229$ & 30 & 53,2523 & 28 & High P \\
\hline 48 & 1159 & 1.64 & $\mathrm{a} 00000230$ & 30 & & 28 & \\
\hline 49 & 1206 & 1.66 & $\mathrm{a} 00000231$ & 35 & 50,3792 & 28 & High P \\
\hline 50 & 1165 & 1.63 & $\mathrm{a} 00000232$ & 30 & & 28 & \\
\hline 51 & 1207 & 1.66 & $\mathrm{a} 00000233$ & 30 & 55,3106 & 28 & High P \\
\hline 52 & 1259 & 1.61 & $\mathrm{a} 00000234$ & 30 & & 28 & \\
\hline 53 & 1019 & 1.43 & $\mathrm{a} 00000235$ & 30 & & 28 & \\
\hline 54 & 1051 & 1.55 & $\mathrm{a} 00000236$ & 30 & & 28 & \\
\hline 55 & 1097 & 1.58 & $\mathrm{a} 00000237$ & 35 & 49,7875 & 28 & High P \\
\hline 56 & 1048 & 1.62 & $\mathrm{a} 00000238$ & 30 & 32,7013 & 28 & \\
\hline 57 & 1086 & 1.57 & $\mathrm{a} 00000239$ & 30 & 52,2544 & 28 & High P \\
\hline 58 & 1128 & 1.57 & $\mathrm{a} 00000240$ & 30 & & 28 & \\
\hline 59 & 1054 & 1.58 & $\mathrm{a} 00000241$ & 30 & & 28 & \\
\hline 60 & 1090 & 1.60 & $\mathrm{a} 00000242$ & 30 & 45,0207 & 28 & High P \\
\hline 61 & 1140 & 1.61 & $\mathrm{a} 00000243$ & 30 & & 28 & \\
\hline 62 & 1057 & 0.11 & $\mathrm{a} 00000308$ & 30 & 103,2932 & 25 & \\
\hline 63 & 1263 & 0.11 & $\mathrm{a} 00000309$ & 30 & 165,9728 & 25 & \\
\hline 64 & 977 & 0.23 & $\mathrm{a} 00000310$ & 40 & & 25 & \\
\hline 65 & 1149 & 0.20 & $\mathrm{a} 00000311$ & 30 & 81,5249 & 25 & \\
\hline 66 & 1304 & 0.17 & $\mathrm{a} 00000312$ & 30 & 43,152 & 25 & \\
\hline 67 & 1110 & 1.29 & $\mathrm{a} 00000313$ & 40 & & 25 & \\
\hline 68 & 943 & 2.26 & $\mathrm{a} 00000316$ & 35 & & 26 & \\
\hline 69 & 1299 & 1.22 & a00000334 & 25 & 40,4547 & 29 & \\
\hline 70 & 1182 & 1.22 & $\mathrm{a} 00000335$ & 25 & 167,1738 & 29 & \\
\hline 71 & 1096 & 1.22 & $\mathrm{a} 00000336$ & 30 & & 29 & \\
\hline 72 & 1383 & 1.22 & $\mathrm{a} 00000337$ & 25 & 149,6117 & 29 & \\
\hline
\end{tabular}

10

American Institute of Aeronautics and Astronautics 


\begin{tabular}{|c|c|c|c|c|c|c|c|}
\hline 73 & 1235 & 1.22 & $\mathrm{a} 00000338$ & 25 & & 29 & \\
\hline 74 & 1099 & 1.22 & $\mathrm{a} 00000339$ & 30 & & 29 & \\
\hline 75 & 1387 & 1.22 & $\mathrm{a} 00000340$ & 25 & & 29 & \\
\hline 76 & 1228 & 1.22 & $\mathrm{a} 00000341$ & 25 & 39,3955 & 29 & \\
\hline 77 & 1116 & 1.22 & $\mathrm{a} 00000342$ & 35 & & 29 & \\
\hline 78 & 1264 & 3.24 & $\mathrm{a} 00000343$ & 30 & 39,4483 & 29 & \\
\hline 79 & 1243 & 3.24 & $\mathrm{a} 00000344$ & 30 & 37,2183 & 29 & \\
\hline 80 & 1185 & 3.24 & $\mathrm{a} 00000345$ & 35 & & 29 & \\
\hline 81 & 1325 & 3.24 & $\mathrm{a} 00000346$ & 30 & 31,1241 & 29 & \\
\hline 82 & 1204 & 3.24 & $\mathrm{a} 00000347$ & 35 & 85,7406 & 29 & \\
\hline 83 & 1179 & 3.24 & $\mathrm{a} 00000348$ & 35 & & 29 & \\
\hline 84 & 1327 & 3.24 & $\mathrm{a} 00000349$ & 30 & 33,3077 & 29 & \\
\hline 85 & 1259 & 3.24 & $\mathrm{a} 00000350$ & 30 & & 29 & \\
\hline 86 & 1166 & 3.24 & $\mathrm{a} 00000351$ & 40 & & 29 & \\
\hline 87 & 1695 & 0.16 & $\mathrm{a} 00000352$ & 30 & 159,4249 & 29 & \\
\hline 88 & 1351 & 0.16 & $\mathrm{a} 00000353$ & 30 & & 29 & \\
\hline 89 & 980 & 0.16 & $\mathrm{a} 00000354$ & 40 & 77,9251 & 29 & Low T \\
\hline 90 & 2004 & 0.16 & $\mathrm{a} 00000355$ & 25 & & 29 & \\
\hline 91 & 1273 & 0.16 & $\mathrm{a} 00000356$ & 25 & 33,2412 & 29 & \\
\hline 92 & 992 & 0.16 & $\mathrm{a} 00000357$ & 35 & & 29 & \\
\hline 93 & 1975 & 0.16 & $\mathrm{a} 00000358$ & 25 & & 29 & \\
\hline 94 & 1436 & 0.16 & $\mathrm{a} 00000359$ & 25 & & 29 & \\
\hline 95 & 1027 & 0.16 & $\mathrm{a} 00000360$ & 35 & & 29 & \\
\hline
\end{tabular}

The 28 active parameters in studied dataset were kept the same as in the original model. Their bounds were not changes as they showed lower sensitivities than those of the experimental QoI uncertainties and also with the aim to have the smallest parameter modifications of the respective literature recommendations. The final dataset for feasible set construction had 57 experimental QoI and 28 active variables.

\section{B. Feasible set construction}

While $\boldsymbol{H}$ designates prior information, feasible set $\boldsymbol{\Phi}$ summarizes posterior information: all parameter value combinations that satisfy their own bounds and also the QoI included in the dataset bounds. The size and shape of $\boldsymbol{\Phi}$ compared to those of $\boldsymbol{H}$ represent information gained as a result of the B2B-DC analysis. Projection of $\boldsymbol{\Phi}$ on each of the $x$ 's yields the posterior range of the parameter uncertainty. ${ }^{3}$ For the rest of the parameters, the posterior ranges were the same as the prior ones, indicating that the experimental data included in the present analysis did not aid in narrowing down the uncertainty ranges of these parameters individually. However, such an outcome does not necessarily imply no information gain for a given parameter: while the extreme parameter values (bounds) may not change, the feasible set may, and usually does, eliminates some combinations of these parameters with others, which is addressed next. 


\section{Parameter optimization}

While the primary focus of the B2B-DC framework is on prediction over the feasible set, it also supports parameter optimization. ${ }^{47}$ Three sets of optimized model parameters were investigated and inter-compared in the present study. The first approach is LS-H, a (weighted) least-squared fit constraining parameter values to their initially assessed uncertainty ranges, $\boldsymbol{H}$. B2B-DC supports two more refined methods of optimization, ${ }^{47}$ LS-F and $1 \mathrm{~N}-\mathrm{F}$, where the objective is minimized with $x$ 's being constrained to the feasible set $\boldsymbol{\Phi}$. The three problems are easily expressed as mathematical optimizations. The LS methods minimize the familiar sum of weighted least-squared deviations between the surrogate model prediction and the reported measured value, $y_{e}$. The difference lies in where the search takes place: LS-H considers all of $\mathrm{H}$ while LS-F restricts the search to $\mathrm{F}$,

$$
\begin{array}{ll}
\text { LS-H : } & \min _{x \in \mathrm{H}} \sum_{e} w_{e}\left[M_{e}(x)-y_{e}\right]^{2} \\
\text { LS-F : } & \min _{x \in \mathrm{F}} \sum_{e} w_{e}\left[M_{e}(x)-y_{e}\right]^{2}
\end{array}
$$

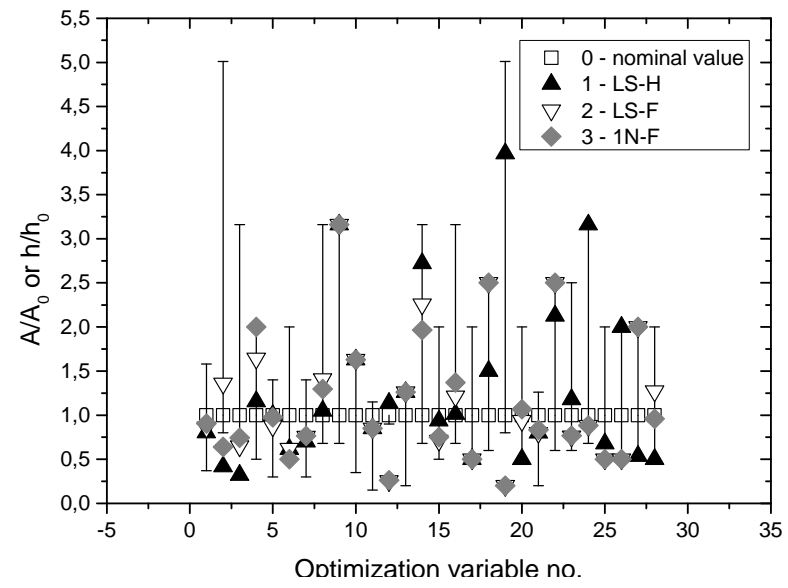

Figure 2. Ratios of optimal to initial values for 28 preexponential factors of rate coefficients obtained with methods LS-H, LS-F and 1N-F. Error bars indicate the specified variable ranges.

By contrast, the $1 \mathrm{~N}-\mathrm{F}$ problem treats the nominal parameter vector, the starting set of parameter values $\left(x_{0}=0\right)$, as "preferred". As we have shown in previsions sections, this parameter set lies outside the feasible region $\boldsymbol{\Phi}$. The goal of the $1 \mathrm{~N}-\mathrm{F}$ method is to find with least number of changes to $x_{0}$ a parameter vector that is feasible. Mathematically, the one-norm is a well-known approximation to enforce such sparsity, i.e.,

1N-F: $\min _{x \in \mathrm{F}}\left\|x-x_{0}\right\|_{1}$

The LS-F and 1N-F optimizations were performed with the final dataset, as the two methods are designed to work with an existing feasible set. The ratios of optimized to initial values for 28 rate coefficients obtained with

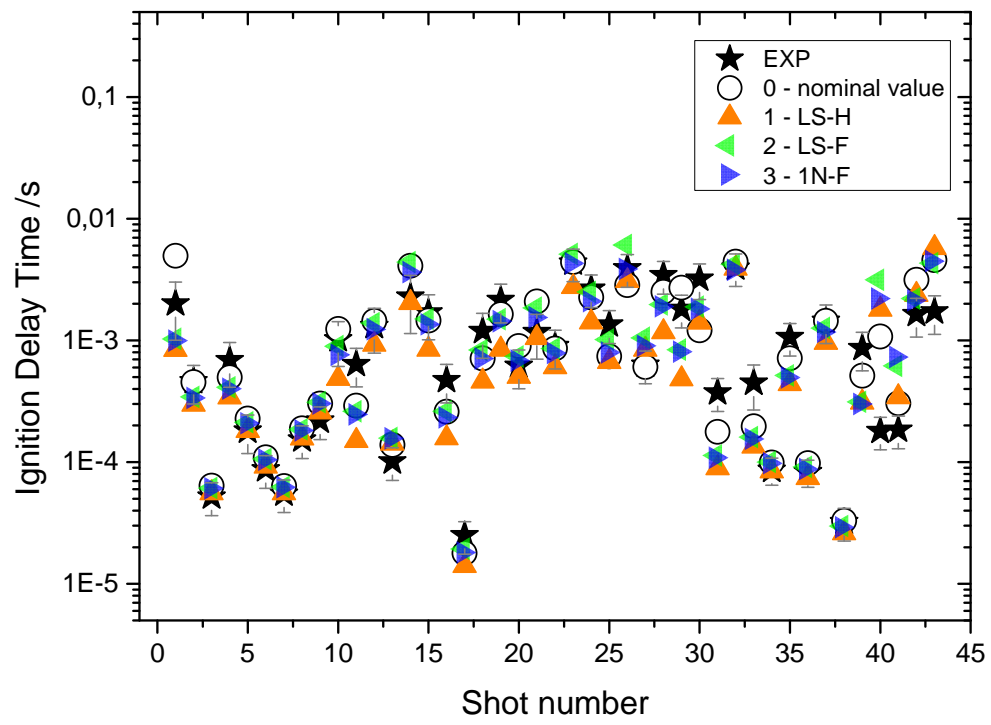

Figure 3. Optimal model predictions of ignition delay times using optimization methods LS-H, LS-F and $1 \mathrm{~N}-\mathrm{F}$. Nominal value modeling with original model. methods LS-F and $1 \mathrm{~N}-\mathrm{F}$ are shown in Fig. 2

Inspection of the results highlights several features. All optimization methods result in parameter sets that produce a better agreement with experiment than the original model, composed of literature recommendations. The LS-H optimization, constrained only to the prior uncertainty ranges of parameters, results in the lowest average deviation, as expected, but at the expense of violating uncertainty bounds of 13 experimental QoI.

The average deviation produced by LS-F is larger but not significantly than that of LS-H. The $1 \mathrm{~N}-\mathrm{F}$ method gives a larger average deviation, yet it changes the least number of variables. The LS-F and 1N-F optimization methods, with additional constraints to the QoI uncertainties, do not violate 


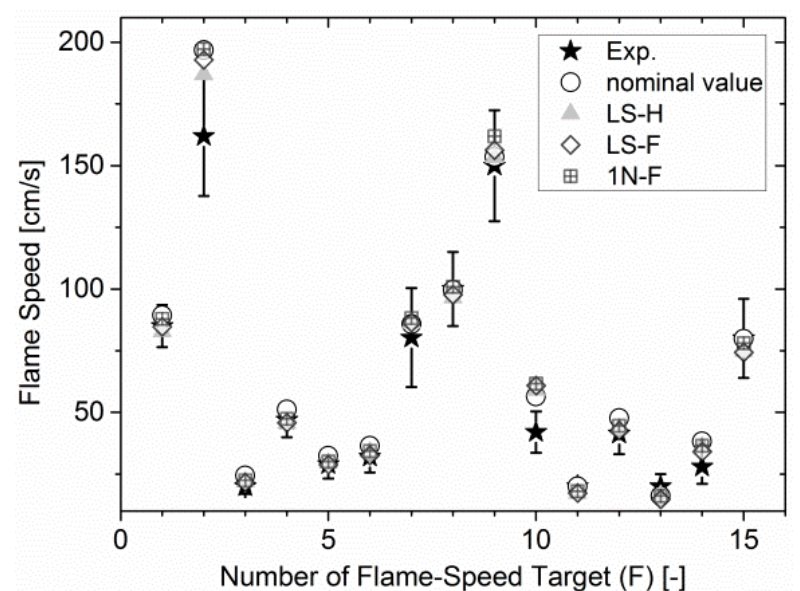

Figure 4. Optimal model predictions of laminar flame speed using optimization methods LS-H, LS-F and 1N-F. Nominal value - modeling with original model.

any of the QoI bounds by design unlike to the LS-H. That demonstrates the main difference between two approaches: LS-H optimization can be identified rater as a fitting, Fig. 3 and 4.

The better agreement of the optimized model with the selected experimental data should not be presumed, however, to be the final step of the analysis. While the obtained optimized parameter values can serve as an indicator for parameter inadequacy, the direction of possible parameter modifications can be further revealed from analysis of correlations. Such correlations are displayed in Figures 5 and 6, which display results of random sampling of the feasible set. Figure 5 depicts correlation plots of selected model parameters. The displayed results illustrate trends in parameter distributions and correlations between different reaction rate coefficients. Figure 6 presents similar information but includes also correlations among QoI and correlations among model parameters and QoI. These diagrams highlight the "bound-to-bound" concept and demonstrate the influence of experimental data on the active parameter distributions. Noteworthy is the fact that the feasible-set regions, identified by the sampled points marked in blue, are not centered for some QoI and for some parameters. If the prior information were exactly

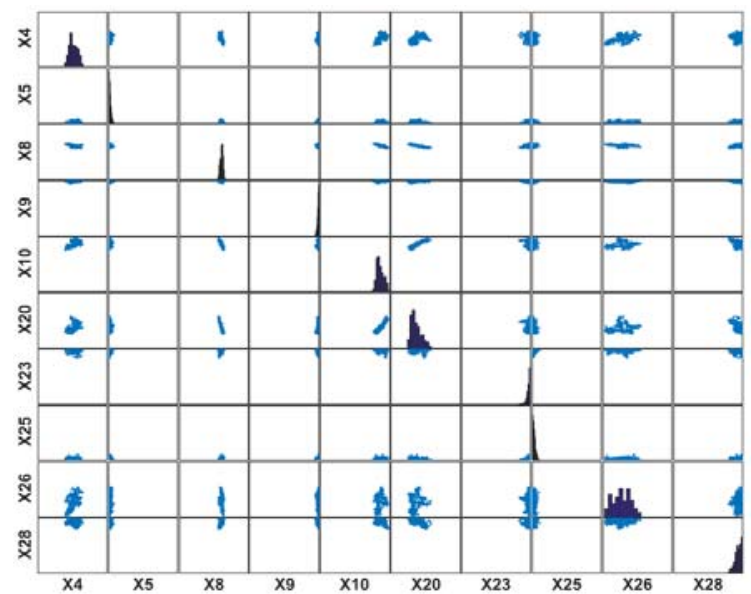

Figure 5. Correlation plots of selected parameters (Table 1). The axis intervals represent their uncertainty ranges, $[-1,1]$. correct, both the experiment and the model, than we should have the feasible set centered at the nominal values of parameters and measured QoI, i.e., located in the center of each diagram. The fact that in many cases the feasible set is "pushed" to the boundaries indicates the presence of either systematic errors in experiments or bias in the model. The information provided in the analysis thus helps to focus on the issues (experiments, parameters, model) that need immediate attention in moving toward a more predictive model.

Finally, the comparison of model predictions obtained with the original and optimized mechanisms for the experimental QoI is shown in Fig.7. As can be seen in Fig.7, the model optimized on the feasible set improves the experimental reproduction not only for QoI of the dataset but also for those not included in the B2B-DC analysis, Fig. $7 \mathrm{~b}$. This is in contrast to the results obtained with the model optimized on the entire hypercube $H$, which does not describe correctly neither the trend nor the values of the experimental observations. This demonstrates the benefits of optimization methods LS-F and 1N-F and generally of the B2B-DC methodology in comparison to the "conventional" optimization, LS-H. 


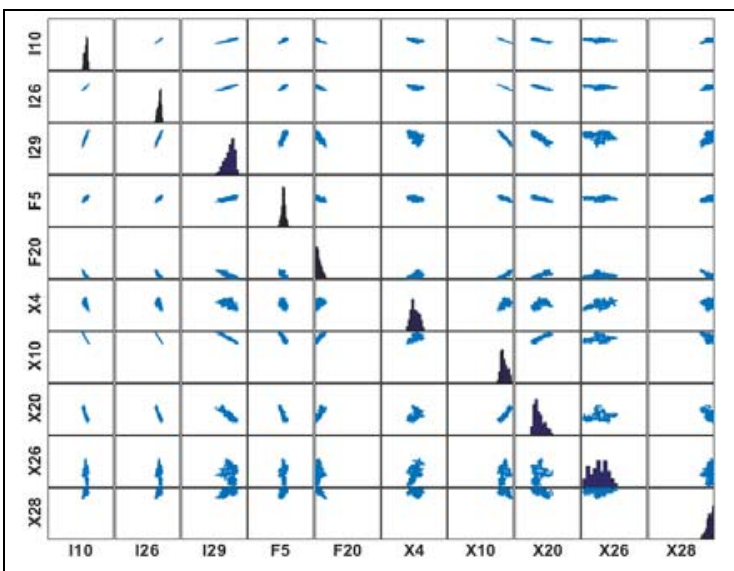

Figure 6. Correlation plots of selected active parameters $(\mathrm{X})$ and of selected ignition-delay (I) and flame-speed (F) QoIs. The QoI axis intervals represent their respective uncertainty ranges, [Le, Ue].

\section{Conclusions}

An optimization-based framework B2B-DC of an automated data-centric infrastructure, Process Informatics Model (PrIMe) was applied to the syngas reaction mechanism analysis with the aim to test the PrIMe software facilities. For this purpose, a dataset was constructed based on pertinent experimental observations, chemical-kinetics model, and the associated uncertainties. The 118 experimental Quantities of Interest (QoI) were selected through evaluation of ignition delay time and laminar flame speed uncertainties. The composed dataset was subjected to consistency analysis. One outcome of the analysis was identification of a set of experimental QoI that were most difficult or impossible to match with the model; they were removed from the dataset for future investigation. The final consistent dataset with 57 experimental QoI and 28 active variables was used for model optimization on the feasible parameter set. The optimized syngas models produced with B2B-DC framework demonstrated an improved agreement with the dataset QoI, as well as with experimental measurements not included in the analysis. The obtained optimized parameter values indicated parameter inadequacy, and the correlation analysis highlighted the direction of possible parameter modifications and model improvement. The algorithm of an application of the PrIme Data Collaboration module must be investigated further.

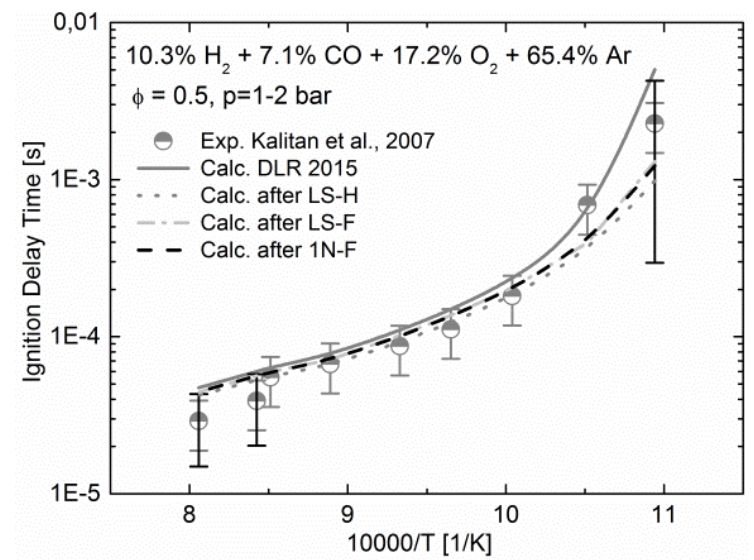

a

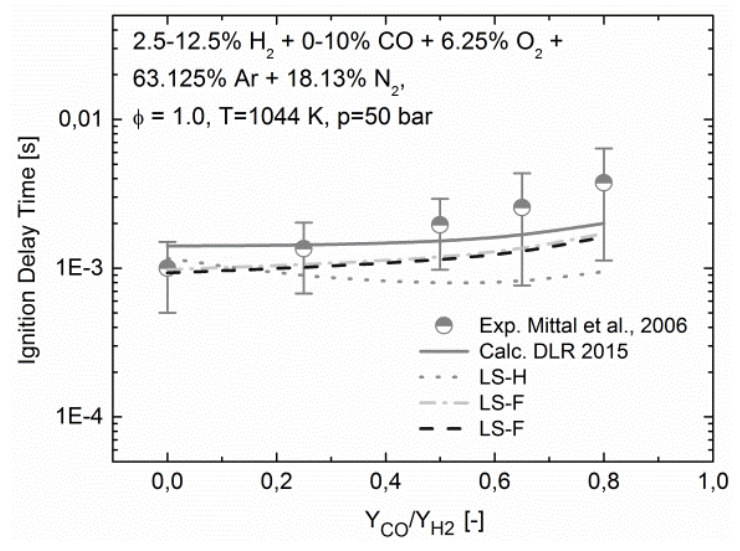

b

Figure 7. Optimal model predictions of ignition delay times using optimization methods LS-H, LS-F and $1 N-F$. The bars indicate the specified data and changed ranges. a) experimental data ${ }^{32}$ included in the dataset; b) experimental data ${ }^{48}$ not included in the dataset.

\section{Acknowledgments}

The work at UC Berkeley was supported by the U.S. Department of Energy, National Nuclear Security Administration, under Award Number DE-NA0002375. 


\section{References}

${ }^{1}$ Frenklach, M., "PrIMe," URL: http://primekinetics.org.

${ }^{2}$ Feeley, R., Seiler, P., Packard, A., and Frenklach, M., "Consistency of a Reaction Dataset," The Journal of Physical Chemistry A, Vol. 108, No. 44, 2004, pp. 9573-9583.

${ }^{3}$ Frenklach, M., Packard, A., Seiler, P., and Feeley, R., "Collaborative Data Processing in Developing Predictive Models of Complex Reaction Systems," International Journal of Chemical Kinetics, Vol. 36, No. 1, 2004, pp. 57-66.

${ }^{4}$ Russi, T., Packard, A., Feeley, R., and Frenklach, M., "Sensitivity Analysis of Uncertainty in Model Prediction," The journal of physical chemistry. A, Vol. 112, No. 12, 2008, pp. 2579-2588.

${ }^{5}$ Russi, T., Packard, A., and Frenklach, M., "Uncertainty Quantification: Making Predictions of Complex Reaction Systems Reliable," Chemical Physics Letters, Vol. 499, 1-3, 2010, pp. 1-8.

${ }^{6}$ Frenklach, M., Packard, A., and Feeley, R., "Optimization of Reaction Models with Solution Mapping," Modeling of Chemical Reactions, edited by R. W. Carr, 1st ed., Elsevier, Amsterdam, 2007, pp. 243-293.

${ }^{7}$ Seiler, P., Frenklach, M., Packard, A., and Feeley, R., "Numerical Approaches for Collaborative Data Processing," Optimization and Engineering, Vol. 7, No. 4, 2006, pp. 459-478.

${ }^{8}$ Frenklach, M., "Transforming Data into Knowledge-Process Informatics for Combustion Chemistry," Proceedings of the Combustion Institute, Vol. 31, No. 1, 2007, pp. 125-140.

${ }^{9}$ Slavinskaya, N. A., Riedel, U., Dworkin, S. B., and Thomson, M. J., "Detailed Numerical Modeling of PAH Formation and Growth in Non-premixed Ethylene and Ethane Flames," Combustion and Flame, Vol. 159, No. 3, 2012, pp. 979-995.

${ }^{10}$ Olm, C., Zsély, I. G., Varga, T., Curran, H. J., and Turányi, T., “Comparison of the Performance of Several Recent Syngas Combustion Mechanisms," Combustion and Flame, Vol. 162, No. 5, 2015, pp. 1793-1812.

${ }^{11}$ Atkinson, R., Baulch, D. L., Cox, R. A., Crowley, J. N., Hampson, R. F., Hynes, R. G., Jenkin, M. E., Rossi, M. J., and Troe, J., "Evaluated Kinetic and Photochemical Data for Atmospheric Chemistry: Volume I - Gas Phase Reactions of $\mathrm{O}_{\mathrm{x}}, \mathrm{HO}_{\mathrm{x}}$, $\mathrm{NO}_{\mathrm{x}}$ and $\mathrm{SO}_{\mathrm{x}}$ Species," Atmospheric Chemistry and Physics, Vol. 4, 2004, pp. 1461-1738.

${ }^{12}$ Wang, H., You, X., Joshi, A. V., Davis, S. G., Laskin, A., Egolfopoulos, F., and Law, C. K., "USC Mech Version II. HighTemperature Combustion Reaction Model of $\mathrm{H}_{2} / \mathrm{CO} / \mathrm{C} 1-\mathrm{C} 4$ Compounds," URL: http://ignis.usc.edu/USC_Mech_II.htm.

${ }^{13}$ Miller, J. A., and Melius, C. F., "Kinetic and Thermodynamic Issues in the Formation of Aromatic Compounds in Flames of Aliphatic Fuels," Combustion and Flame, Vol. 91, No. 1, 1992, pp. 21-39.

${ }^{14}$ Kathrotia, T., Fikri, M., Bozkurt, M., Hartmann, M., Riedel, U., and Schulz, C., "Study of the H+O+M Reaction Forming $\mathrm{OH}^{*}$ : Kinetics of $\mathrm{OH}^{*}$ Chemiluminescence in Hydrogen Combustion Systems," Combustion and Flame, Vol. 157, No. 7, 2010, pp. 1261-1273.

${ }^{15}$ Baulch, D. L., "Evaluated Kinetic Data for Combustion Modeling: Supplement II," Journal of Physical and Chemical Reference Data, Vol. 34, No. 3, 2005, p. 757.

${ }^{16}$ Troe, J., "Detailed modeling of the temperature and pressure dependence of the reaction $\mathrm{H}+\mathrm{O}_{2}(+\mathrm{M}) \rightarrow \mathrm{HO}_{2}(+\mathrm{M})$," Proceedings of the Combustion Institute, Vol. 28, No. 2, 2000, pp. 1463-1469.

${ }^{17}$ Cohen, N., Westberg, K. R., "Chemical Kinetic Data Sheets for High-Temperature Chemical Reactions,” Journal of Physical and Chemical Reference Data, Vol. 12, No. 3, 1983, pp. 531-590.

${ }^{18}$ Zsély, I. G., Zádor, J., and Turányi, T., "Uncertainty Analysis of Updated Hydrogen and Carbon Monoxide Oxidation Mechanisms," Proceedings of the Combustion Institute, Vol. 30, No. 1, 2005, pp. 1273-1281.

${ }^{19}$ Sun, H., Yang, S. I., Jomaas, G., and Law, C. K., "High-pressure Laminar Flame Speeds and Kinetic Modeling of Carbon Monoxide/Hydrogen Combustion," Proceedings of the Combustion Institute, Vol. 31, No. 1, 2007, pp. 439-446.

${ }^{20}$ Troe, J. "Predictive Possibilities of Unimolecular Rate Theory," The Journal of Physical Chemistry, Vol. 83, No. 1, 1979, pp. $114-126$.

${ }^{21}$ Li, J., Zhao, Z., Kazakov, A., Chaos, M., Dryer, F. L., and Scire, J. J., “A Comprehensive Kinetic Mechanism for CO, $\mathrm{CH}_{2} \mathrm{O}$, and $\mathrm{CH}_{3} \mathrm{OH}$ Combustion," International Journal of Chemical Kinetics, Vol. 39, No. 3, 2007, pp. 109-136.

${ }^{22}$ Davidson, D. F., and Hanson, R. K., "Interpreting Shock Tube Ignition Data," WSSCI Fall 2003 Meeting, University of California at Los Angeles, 2003.

${ }^{23}$ Petersen, E. L., and Hanson, R. K., "Nonideal Effects Behind Reflected Shock Waves in a High-pressure Shock Tube,” Shock Waves, Vol. 10, No. 6, 2001, pp. 405-420. 
${ }^{24}$ Petersen, E. L., Rickard, M. J. A., Crofton, M. W., Abbey, E. D., Traum, M. J., and Kalitan, D. M., “A Facility for Gas- and Condensed-phase Measurements Behind Shock Waves," Measurement Science and Technology, Vol. 16, No. 9, 2005, pp. $1716-1729$.

${ }^{25}$ Petersen, E. L., and Hanson, R. K., "Measurement of Reflected-shock Bifurcation Over a Wide Range of Gas Composition and Pressure," Shock Waves, Vol. 15, No. 5, 2006, pp. 333-340.

${ }^{26}$ Dryer, F. L., and Chaos, M., "Ignition of Syngas/Air and Hydrogen/Air Mixtures at Low Temperatures and High Pressures: Experimental Data Interpretation and Kinetic Modeling Implications,” Combustion and Flame, Vol. 152, 1-2, 2008, pp. 293-299.

${ }^{27}$ Davidson, D. F., and Hanson, R. K., "Recent Advances in Shock Tube/Laser Diagnostic Methods for Improved Chemical Kinetics Measurements," Shock Waves, Vol. 19, No. 4, 2009, pp. 271-283.

${ }^{28}$ Ihme, M., "On the Role of Turbulence and Compositional Fluctuations in Rapid Compression Machines: Autoignition of Syngas Mixtures," Combustion and Flame, Vol. 159, No. 4, 2012, pp. 1592-1604.

${ }^{29}$ Urzay, J., Kseib, N., Davidson, D. F., Iaccarino, G., and Hanson, R. K., "Uncertainty-quantification Analysis of the Effects of Residual Impurities on Hydrogen-oxygen Ignition in Shock Tubes,” Combustion and Flame, Vol. 161, No. 1, 2014, pp. 1-15.

${ }^{30}$ Mansfield, A. B., and Wooldridge, M. S., "High-pressure Low-temperature Ignition Behavior of Syngas Mixtures," Combustion and Flame, Vol. 161, No. 9, 2014, pp. 2242-2251.

${ }^{31}$ Grogan, K. P., and Ihme, M., "Weak and Strong Ignition of Hydrogen/Oxygen Mixtures in Shock-tube Systems," Proceedings of the Combustion Institute, Vol. 35, No. 2, 2015, pp. 2181-2189.

${ }^{32}$ Kalitan, D. M., Mertens, J. D., Crofton, M. W., and Petersen, E. L., "Ignition and Oxidation of Lean CO/H2 Fuel Blends in Air,” Journal of Propulsion and Power, Vol. 23, No. 6, 2007, pp. 1291-1301.

${ }^{33}$ Petersen, E. L., Kalitan, D. M., Barrett, A. B., Reehal, S. C., Mertens, J. D., Beerer, D. J., Hack, R. L., and McDonell, V. G., "New Syngas/Air Ignition Data at Lower Temperature and Elevated Pressure and Comparison to Current Kinetics Models," Combustion and Flame, Vol. 149, 1-2, 2007, pp. 244-247.

${ }^{34}$ Mertens, J. D., Kalitan, D. M., Barrett, A. B., and Petersen, E. L., "Determination of the Rate of $\mathrm{H}+\mathrm{O}_{2}+\mathrm{M} \rightarrow \mathrm{HO}_{2}+\mathrm{M}\left(\mathrm{M}=\mathrm{N}_{2}\right.$, $\mathrm{Ar}, \mathrm{H}_{2} \mathrm{O}$ ) from Ignition of Syngas at Practical Conditions," Proceedings of the Combustion Institute, Vol. 32, No. 1, 2009, pp. 295-303.

${ }^{35}$ Herzler, J., and Naumann, C., "Shock Tube Study of the Ignition of Lean $\mathrm{CO} / \mathrm{H}_{2}$ Fuel Blends at Intermediate Temperatures and High Pressure," Combustion Science and Technology, Vol. 180, 10-11, 2008, pp. 2015-2028.

${ }^{36}$ Kéromnès, A., Metcalfe, W. K., Heufer, K. A., Donohoe, N., Das, A. K., Sung, C.-J., Herzler, J., Naumann, C., Griebel, P., Mathieu, O., Krejci, M. C., Petersen, E. L., Pitz, W. J., and Curran, H. J., “An Experimental and Detailed Chemical Kinetic Modeling Study of Hydrogen and Syngas Mixture Oxidation at Elevated Pressures," Combustion and Flame, Vol. 160, No. 6, 2013, pp. 995-1011.

${ }^{37}$ Bouvet, N., Chauveau, C., Gökalp, I., and Halter, F., "Experimental Studies of the Fundamental Flame Speeds of Syngas $\left(\mathrm{H}_{2} / \mathrm{CO}\right) /$ Air Mixtures," Proceedings of the Combustion Institute, Vol. 33, No. 1, 2011, pp. 913-920.

${ }^{38}$ Esposito, G., and Chelliah, H. K., "Effect of Binary Diffusion and Chemical Kinetic Parameter Uncertainties in Simulations of Premixed and Non-premixed Laminar Hydrogen Flames," Combustion and Flame, Vol. 159, 2012, pp. 3522-3529.

${ }^{39}$ Goswami, M., Derks, S. C., Coumans, K., Slikker, W. J., Andrade Oliveira, M. H. de, Bastiaans, R. J., Luijten, C. C., Goey, L. P. H. de, and Konnov, A. A., "The Effect of Elevated Pressures on the Laminar Burning Velocity of Methane+Air Mixtures," Combustion and Flame, Vol. 160, No. 9, 2013, pp. 1627-1635.

${ }^{40}$ Egolfopoulos, F. N., Hansen, N., Ju, Y., Kohse-Höinghaus, K., Law, C. K., and Qi, F., “Advances and Challenges in Laminar Flame Experiments and Implications for Combustion Chemistry," Progress in Energy and Combustion Science, Vol. 43, 2014, pp. 36-67.

${ }^{41}$ Krejci, M. C., Mathieu, O., Vissotski, A. J., Ravi, S., Sikes, T. G., Petersen, E. L., Kérmonès, A., Metcalfe, W., and Curran, H. J., "Laminar Flame Speed and Ignition Delay Time Data for the Kinetic Modeling of Hydrogen and Syngas Fuel Blends," Journal of Engineering for Gas Turbines and Power, Vol. 135, No. 2, 2013, p. 021503.

${ }^{42}$ Natarajan, J., Lieuwen, T., and Seitzman, J., "Laminar Flame Speeds of $\mathrm{H}_{2} / \mathrm{CO}$ Mixtures: Effect of $\mathrm{CO}_{2}$ Dilution, Preheat Temperature, and Pressure," Combustion and Flame, Vol. 151, 1-2, 2007, pp. 104-119.

${ }^{43}$ Hassan, M. I., Aung, K. T., and Faeth, G. M., "Properties of Laminar Premixed CO/H/Air Flames at Various Pressures," Journal of Propulsion and Power, Vol. 13, No. 2, 1997, pp. 239-245.

${ }^{44}$ Natarajan, J., Kochar, Y., Lieuwen, T., and Seitzman, J., "Pressure and Preheat Dependence of Laminar Flame Speeds of $\mathrm{H}_{2} / \mathrm{CO} / \mathrm{CO}_{2} / \mathrm{O}_{2} / \mathrm{He}$ Mixtures," Proceedings of the Combustion Institute, Vol. 32, No. 1, 2009, pp. 1261-1268.

${ }^{45}$ Kee, R. J., Rupley, F. M., and Miller, J. A., "CHEMKIN-II: A FORTRAN chemical kinetics package for the analysis of gasphase chemical kinetics," SAND89-8009B, UC-706; Sandia National Laboratories: Albuquerque, NM, 1993. 
${ }^{46}$ Kintech Lab Ltd., "Chemical Workbench®,” Software Package, URL: http://www.kintechlab.com/.

${ }^{47}$ You, X., Russi, T., Packard, A., and Frenklach, M., "Optimization of Combustion Kinetic Models on a Feasible Set," Proceedings of the Combustion Institute, Vol. 33, No. 1, 2011, pp. 509-516.

${ }^{48}$ Mittal, G., Sung, C.-J., and Yetter, R. A., "Autoignition of $\mathrm{H}_{2} / \mathrm{CO}$ at Elevated Pressures in a Rapid Compression Machine," International Journal of Chemical Kinetics, Vol. 38, No. 8, 2006, pp. 516-529.

${ }^{50}$ Karkach, S. P., Osherov, V. I., "Ab Initio Analysis of the Transition States on the Lowest Triplet $\mathrm{H}_{2} \mathrm{O}_{2}$ Potential Surface," The Journal of Chemical Physics, Vol. 110, 1999, pp. 11918-11927. 